\title{
Nonlinearity of the Rock Joint Shear Strength
}

\author{
Y. F. Wei, ${ }^{a, 1}$ W. X. Fu, ${ }^{\text {b }}$ and D. X. Nie ${ }^{a}$ \\ ${ }^{\text {a }}$ State Key Laboratory of Geohazard Prevention and Geoenvironment Protection, Chengdu University \\ of Technology, Chengdu, China \\ ${ }^{\mathrm{b}}$ State Key Laboratory of Hydraulic and Mountain River Engineering, Sichuan University, Chengdu, \\ China \\ ${ }^{1}$ weiyufeng@cdut.edu.cn
}

The triaxial testing for irregular or unfilled rock joints was conducted on the rock mechanics test system (MTS). A series of axial failure stresses under different confining pressures applied to the same specimen was continuously acquired on MTS. The corresponding normal and shear stresses acting on the rock joint plane were calculated in terms of LEFM. The Mohr-Coulomb (MC) shear strength parameters of each specimen could be determined by linear regression analysis. Thirteen specimens were taken from the dam site drill rock cores of a hydropower station. The scatter of points plotted for all test results in the normal and shear stress space exhibits obvious nonlinearity. Test results show that it would be more convenient to describe the shear strength of rock joints in the nonlinear form. The comparison and discussion of three function fittings proved that the well-known Barton criterion was more appropriate for describing the shear strength of rock joints.

Keywords: rock joint, shear strength, mechanics test system, triaxial testing, function fitting, Barton criterion.

Introduction. The irregular and unfilled rock joints are quite widespread discontinuities in the rock mass [1-5]. In addition to straightforward shear testing of discontinuities of this type used by many researchers, an empirical formula for describing the shear strength of rock joints has been proposed. Considering the nonlinear characteristic of the jointed rocks and the effect of the intermediate principal stress on the strength behavior, authors [6] modified the nonlinear form of the Mohr strength criterion. Other researchers also made contribution to the nonlinear shear strength features of the jointed rocks on the basis of the previous researches $[7,8]$.

A consistent concept accepted in the field of rock mechanics is that the shear strength of the rock joint mainly depends on its roughness, hardness and mother rock type and has a nonlinear feature related to stress levels. Based on the shear strength results obtained by the simply-direct shear testing for the structural plane specimens, Barton put forward a nonlinear empirical formula for estimating the peak shear strength of the irregular and non-filled rock joints [9]. The Barton criterion has been wildly accepted in the field of rock mechanics and applied to many rock engineering projects.

Generally, the in-situ direct testing is a more reliable approach to evaluating the shear strength of the rock joints [10-13]. However, due to the difficulties and high costs of the in-situ testing, the laboratory direct shear test method proposed by the International Society for Rock Mechanics Commission (ISRM) is often employed to investigate the shear strength of the rock joints [14]. The test method proposed by the ISRM, which comprises the single and multiple specimen methods, and the corresponding experimental procedure are described in detail by Barton [15]. In addition, Barla et al. developed a new direct shear testing apparatus for testing the strength of rock joints as well [16]. Tatone and some other researchers also provided the quantitative description for the surface characteristics of the rock structural plane using some advanced tools [17]. Some new shear strength criterions 
were proposed for the rock joints together with the surface roughness features measured in the laboratory shear tests $[18,19]$. Jafari et al. [20] analyzed the variation of the shear strength of the rock joints under the cyclic loading excitation, and according to their experimental results, some mathematical models were developed for evaluating the shear strength under the cyclic loading conditions.

In general, the study on the shear strength of the rock mass or rock joints was focused on the theoretical analysis for determinating the strength parameter value and the development of the advanced instrument. However, only few studies were dedicated to the analysis of the experimental method. Considering the reliability of the experimental results, the strength parameters of the rock joint obtained by the direct shear testing are considered the most lucrative. Nevertheless, it is extremely difficult to guarantee the consistency of such parameters as the roughness and hardness of the rock joint specimens. In order to accurately evaluate the strength parameters of the rock joints, the triaxial testing method is presented in this paper. The proposed method would be beneficial for investigating the linear or nonlinear strength parameters of the structural planes.

The triaxial tests of the rock joints in this study were conducted using the rock mechanics test system (MTS). The experimental procedure is similar to the general triaxial testing for the intact rock specimens. The number of the tested specimens is thirteen in total. These specimens were taken from the dam-site drilled rock cores of the Maerdang hydropower station in the upstream of the Yellow river. During the triaxial testing via the MTS, a series of axial failure stresses and confining pressures applied to the same specimen were continuously acquired. Then within framework of the linear elastic fracture mechanics (LEFM) the normal and shear stress components on the rock joint plane are calculated. Herein, the MC shear strength parameters of each specimen can be determined through linear fitting. However, the scatter point distribution of the test results for all specimens showed an obviously nonlinear feature in the normal and shear stress space. Therefore, it was considered more appropriate to describe the shear strength of the tested rock joint specimens in a nonlinear form. On the basis of comparative analysis of three function fittings for the scatter points in the normal and shear stress space, the well-known Barton criterion describing the rock joint shear strength is proved to be appropriate.

1. Experimental Procedure. For the triaxial testing for the rock joints we employed the MTS apparatus with a programmable servo controlling system, which consists of a main test machine, a hydraulic power source system and a digital control system (Fig. 1). The experimental procedure for the rock joint specimen is similar to the general triaxial testing for the intact rock specimen. The axial load was applied gradually until the axial failure stress $\sigma_{1}$ reached a specified confining pressure $\left(\sigma_{2}=\sigma_{3}\right)$. The pressure excitation was gradually increased until the upper part of the rock joint specimen contacted the base plate in the specimen box, or the axial displacement exceeded the reserved displacement space. We thus could attain multiple failure stress pairs of $\left(\sigma_{1}, \sigma_{3}\right)$ for each specimen. The strain rate during axial loading was controlled to be $0.01 \mathrm{~mm} / \mathrm{s}$. We carried out testing for a total of thirteen specimens of rock joints taken from the dam-site drilled rock cores of the Maerdang hydropower station located in the upper stream of the Yellow river. The mother rock type of specimens was monzonite. The natural rock joint had completely separated and was irregular, rough, unfilled and unbonded.

The description of the experiments can be reduced to the following:

(1) The height of each rock joint specimen was less than $100 \mathrm{~mm}$ and was roughly two times the diameter. The maximum sliding distance of 10-20 mm was reserved near the top and bottom of each rock joint specimen, respectively (Fig. 2a). Thus, the upper and lower parts of the specimen could freely move under different confining pressures. The triaxial testing would be terminated when the relative sliding displacement of the upper and low parts reached the $10-20 \mathrm{~mm}$ reserved in advance. 


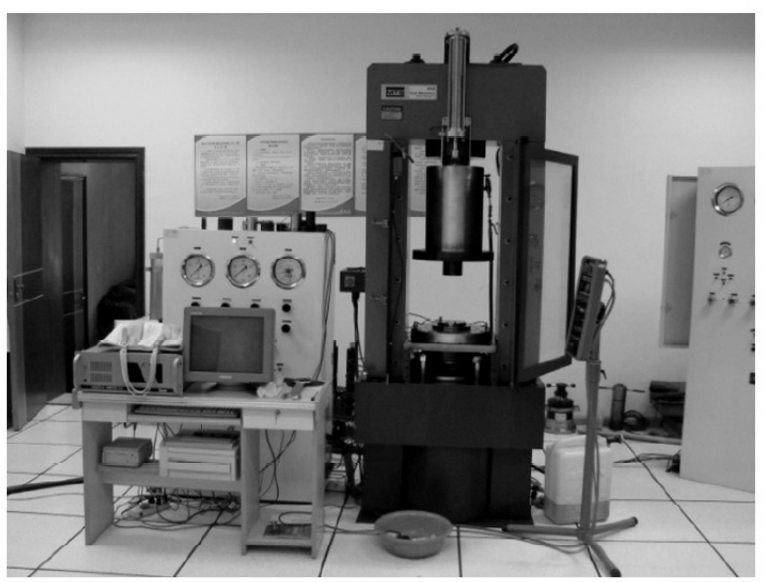

Fig. 1. The rock mechanics test system.

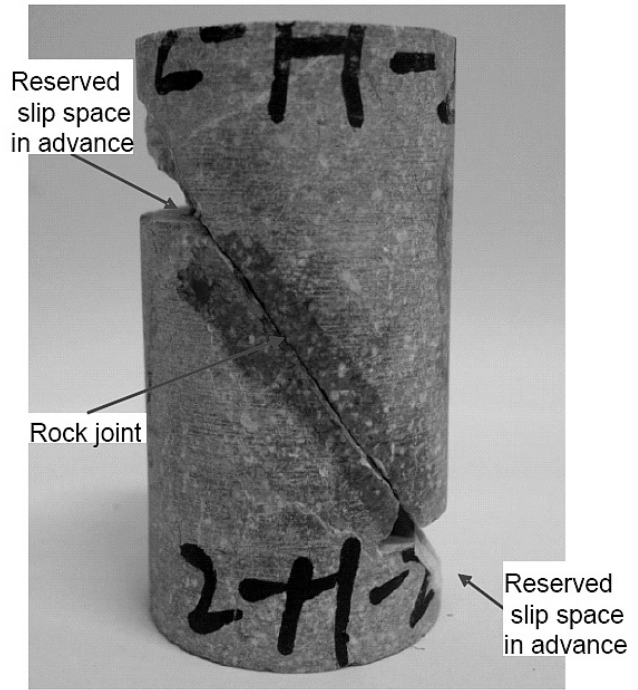

a

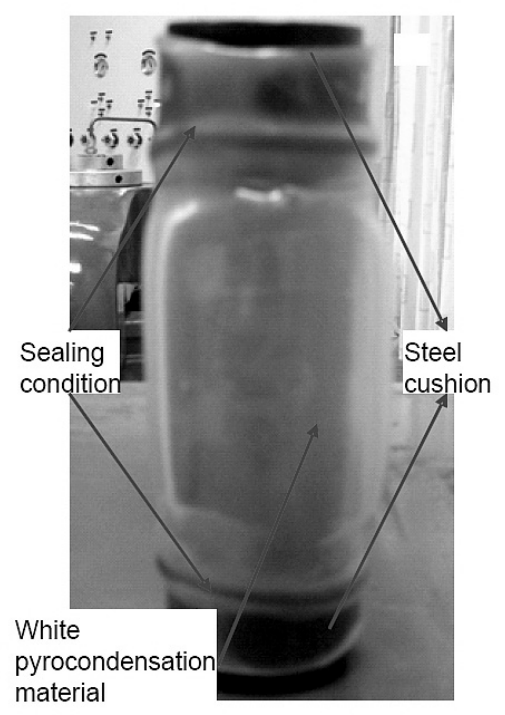

b

Fig. 2. Preparation for the tested specimen: (a) reserving sliding displacement space cut by a diamond saw in advance; (b) wrapping with heat shrink films to prevent hydraulic oil into rock joint fissure.

(2) The confining pressures during the triaxial testing were achieved by increasing the oil pressure in the pressure chamber. The specimens were wrapped with heat shrink films so that each rock joint specimen did not be directly placed into the hydraulic oil. During the wrapping process, the top and bottom blocks of specimen were kept in contact with each other as tightly as possible. After that, the heat shrink films on both ends were heated so that the films shrank and tightened around the surrounding area of the steel caps at two ends of the specimen (Fig. 2b).

The single specimen method [21, 22] was employed in this study. Firstly, the specimen was installed in the triaxial chamber by the conventional triaxial test method. Secondly, the first level of confining pressure was loaded. Thirdly, keeping the confining pressure constant we loaded the first axial pressure until the peak of the stress-strain curve was directly observed through the data acquisition system of MTS. At this point, we kept the first level of axial stress constant and loaded the second level of confining pressure. 


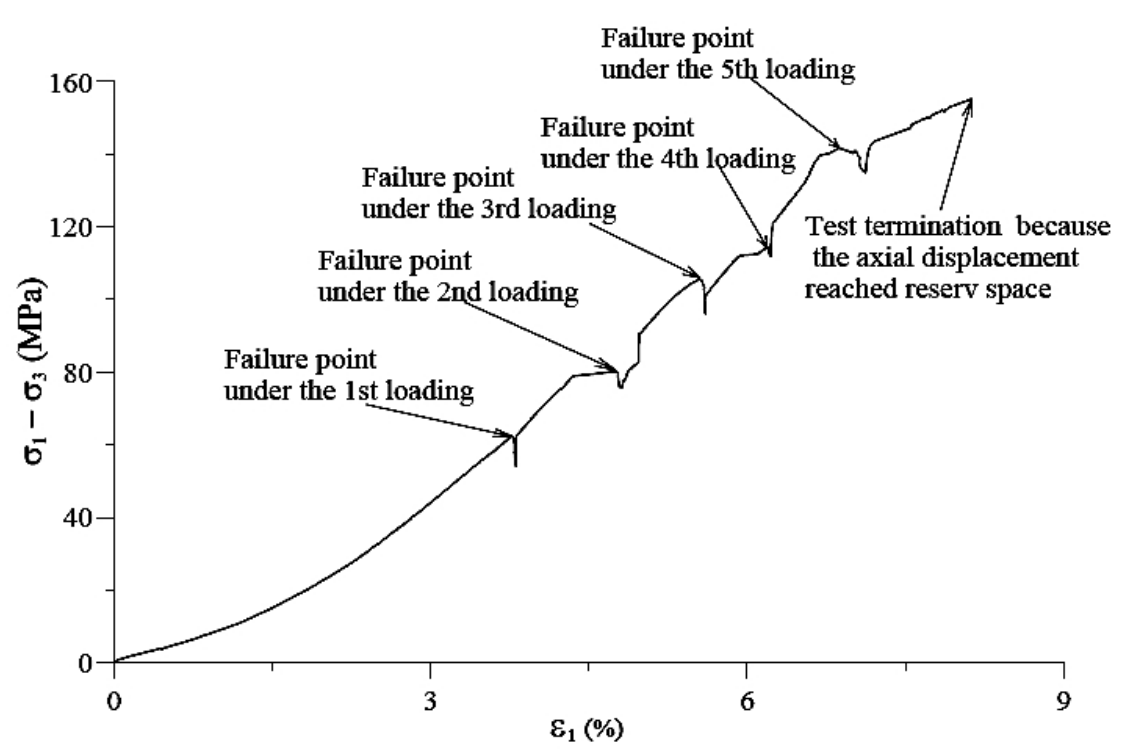

Fig. 3. Loading process of the single specimen test method.

Then we kept the second level of confining pressure constant until the second axial pressure occurred. The repeat loading process for the single specimen test method is described in Fig. 3.

During the triaxial testing for the rock joint specimen, the multiple pairs of the failure stress state $\left(\sigma_{1}, \sigma_{3}\right)$ were recorded during the experiment process. Accordingly, the normal stress and shear stress $\sigma_{n j}$ and $\tau_{j}$ on the structural plane corresponding to the each pair of $\left(\sigma_{1}, \sigma_{3}\right)$ can be calculated by Eqs. (1) and (2), which can be derived in terms of the LEFM theory under the assumption of small strain,

$$
\begin{gathered}
\sigma_{n j}=\frac{\sigma_{1}+\sigma_{3}}{2}-\frac{\sigma_{1}-\sigma_{3}}{2} \cos \beta_{j}, \\
\tau_{j}=\frac{\sigma_{1}-\sigma_{3}}{2} \sin \beta_{j},
\end{gathered}
$$

where $\sigma_{n j}$ and $\tau_{j}$ are the normal stress and the shear stress on the rock joint plane, $\sigma_{1}$ and $\sigma_{3}$ are the axial failure stress (the maximum principal stress) and the confining stress (the minimum principal stress and $\sigma_{2}=\sigma_{3}$ ), and $\beta_{j}$ is the dip angle of the rock joint plane (between the horizontal surface and the rock joint plane).

2. Test Results. The axial failure stresses of the thirteen rock joint specimens could be obtained, as well as the corresponding confining pressures. The normal and shear stresses calculated by Eqs. (1) and (2) can be used for exterminating the frictional angle and cohesion for each rock joint specimen. It should be noted that, theoretically, although only the friction angle existed at the rock joint surface when the two side rock parts of the joint plane were completely separated in natural state, a small amount of the cohesion on the joint plane was still observed during the shearing process. Occurrence of the cohesion was due to the fact that the fissure wall of rock joint was rough and irregular. Figure 4 shows the fresh irregular shear parts on the rock joint plane. A more rough and irregular rock joint specimen would produce a larger shear stress. The clamping effect of a rough and irregular rock joint was more significant, in particular under a high confining pressure. 


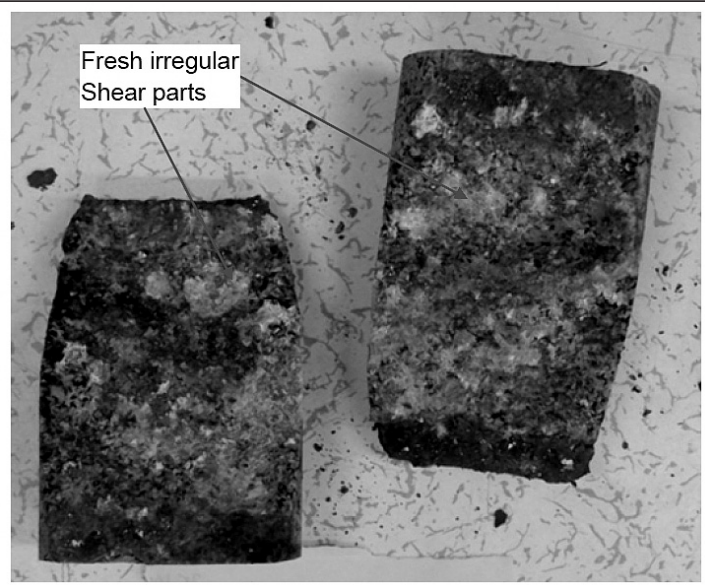

Fig. 4. The fresh irregular shear parts on the rock joint plane.

In fact, the negative cohesions that occurred in some rock joint specimens were also caused by the fresh irregular shear parts on the rock joint plane under a high confining pressure. The rock joint specimens that exhibited the phenomenon of negative cohesions were relatively rare.

\section{Analysis and Discussion.}

3.1. Method of Fitting. Figure 5 showed the scatter points of the normal and shear stress pairs of $\left(\sigma_{n j}, \tau_{j}\right)$ for all specimens. It should be noted that the different shape or color of the dot represented the data pair of $\left(\sigma_{n j}, \tau_{j}\right)$ for each rock joint specimens under different confining pressures. The dip angle of rock joint $\left(\beta_{j}\right)$ and the values of the frictional angle $\varphi_{j}$ and cohesion parameter $c_{j}$ were also given in Fig. 5. Observation for Fig. 5 shows that the shear stress increases with the increase of normal stress and the relation between the normal and shear stresses was obviously nonlinear.

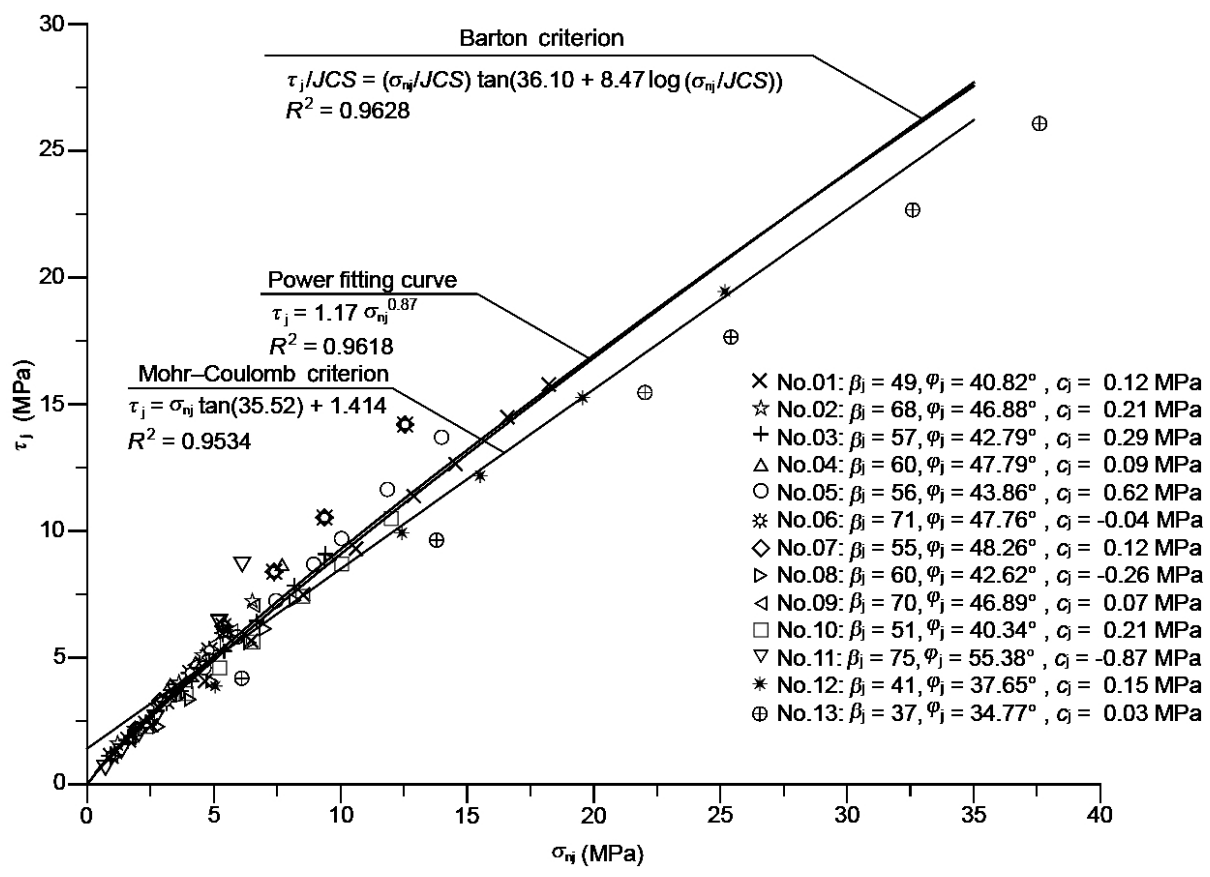

Fig. 5. The scatter point distribution in the $\sigma_{n j}-\tau_{j}$ space and its fitting by three functions. 
In order to reasonably evaluate the relation between the normal and shear stresses of the tested rock joint specimens, the curve-fitting tool in MATLAB was employed to treat the linear Mohr-Coulomb criterion fitting, the power fitting, and the nonlinear Barton criterion fitting. These expressions are given in Eqs (3), (4), and (5), respectively,

$$
\tau_{j}=\tan \varphi_{j} \sigma_{n j}+c_{j},
$$

where $\varphi_{j}$ is the frictional angle of the rock joint (deg) and $c_{j}$ is the cohesion of the rock joint,

$$
\tau_{j}=a \sigma_{n j}^{b}
$$

where $a$ and $b$ are coefficients of the power function,

$$
\tau_{j}=\sigma_{n j} \tan \left(\varphi_{b}+J R C \log \left(\frac{J C S}{\sigma_{n j}}\right)\right) .
$$

Here $\varphi_{b}$ is the basic frictional angle of the rock joint (deg), JRC is the roughness coefficient of the rock joint, and JCS is the compressive strength of the rock joint wall.

When fitting with the power function of Eq. (4), the natural logarithm values of the normal and shear stresses were first calculated. Then Eq. (4) was modified to the linear form as follows:

$$
\ln \tau_{j}=b \ln \sigma_{n j}+\ln a .
$$

The unknown coefficients of Eqs. (3) and (6) can be computed by the least square method. The "general equation" module of the curve fitting tool in MATLAB was chosen to calculate the unknown coefficients in Eq. (5). The "general equation" module allows the user to input any form equation. During the iterative process of calculating the coefficients of the Barton criterion, the initial iterative value should be correctly set for each coefficient, and the initial value should fall within the possible upper and lower limits. The initial iterative input value and the possible upper and lower limits of each parameter should satisfy the physical significance of the Barton criterion. The fitted curves are illustrated in Fig. 5.

3.2 Discussion. The fitted relations by three different approaches showed that the linear $\mathrm{MC}$ criterion fitting is poorer than fitting with the power function or the Barton equation. Especially, the linear MC criterion fitting would overestimate the shear strengths of the rock joints under low normal stress. The curves fitted by a power function and the Barton equation were almost identical and the shear strengths of the rock joints overall demonstrated the nonlinear feature. It also proved the reliability of the triaxial test method. Under a high normal stress the shear strengths estimated by the linear MC criterion, the power function and nonlinear Barton criterion were overestimated. We thus only considered the Barton criterion fitting in the following analysis.

The measured $\sigma_{n j}$ and $\tau_{j}$ were first divided by the fitted JCS-value. Then the relationship of $\sigma_{n j} / J C S$ against $\tau_{j} / J C S$ was plotted (Fig. 6). The results in Fig. 6 were in good agreement with the conclusions conducted by Hoek and Bray [13]. In other words, when $0.01<\sigma_{n j} / J C S<0.3$, the shear strengths of the rock joints estimated by the Barton equation were relatively accurate; but when $\sigma_{n j} / J C S>0.3$, the shear strengths of the rock joints estimated by the Barton equation were overestimated. The protruding parts on the natural and roughness joint surface would be gradually destroyed by the shear stress when the value of the normal stress is high. At the same time, the growth rate of the shear strength would be decreased. This characterization is not consistent with the primary 


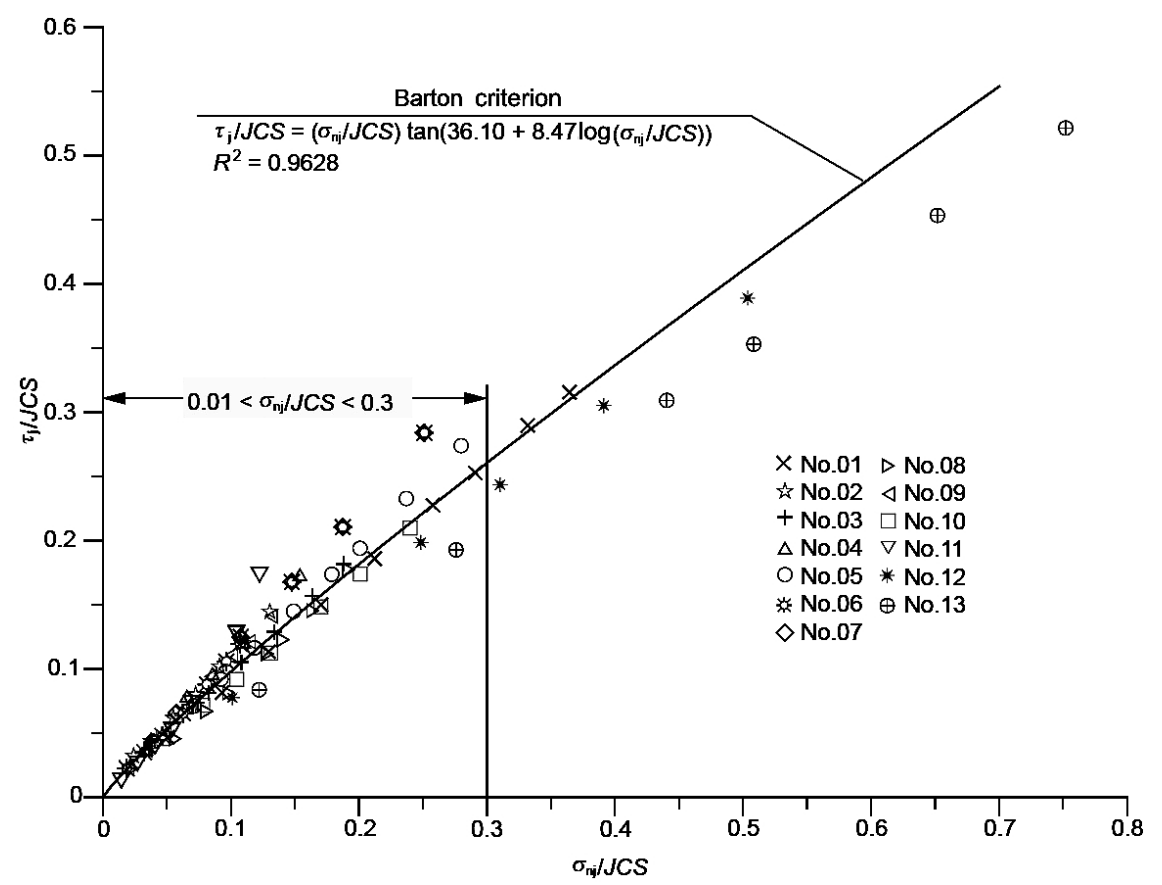

Fig. 6 The reliable range of the rock joint shear strengths estimated by the Barton criterion.

condition of the natural joint surfaces. Hence, under a high normal stress the calculation error would be obvious when the Barton equation was employed. Most of research results showed that the shear strength of the rock joints estimated by the Barton equation would be on the high side when the condition of $\sigma_{n j} / J C S>0.3$ was satisfied. But for the most of the rock engineering projects, such as the slope engineering, this equation was widely used because the value of $\sigma_{n j} / J C S$ normally ranges from 0.01 to 0.3 .

In compliance with Barton's conclusion, the measured shear strength under a high normal stress falls within the range of peak and residual shear strengths when the direct shear testing was repeatedly conducted for the same specimen. In this study, the triaxial testing was continuously conducted under different levels of confining pressure. The shear damage occurred at the locally rough joint surface during the sliding process under the first few levels of confining pressure. Then the shear strength of the joint would be reduced under the next confining pressure tests.

Conclusions. A new test method for determination of the strength parameters of the rock joints is proposed. Some conclusions are summarized as follows:

1. The shear strengths of rock joints were directly determined by using the MST. When controlling the confining pressure at different levels, the normal and shear stresses of rock joints at failure were obtained. The shear strength of the joints related to stress levels were explicitly expressed by the linear MC criterion fitting, the power function fitting and the nonlinear Barton criterion fitting.

2. The presented test method is feasible, while its current deficiencies, such as difficulty of sampling and error caused by different specimens, can be efficiently overcome. The confining pressure can also be designed in terms of the actual buried depth of the in-situ rock joint.

3. Both the power functions fitting, and the nonlinear Barton criterion fitting can produce a more reliable nonlinear shear strength variation related to stress levels than the linear MC criterion fitting. 
Acknowledgments. The present authors thank the State Key Laboratory of Geohazard Prevention and Geoenvironment Protection (Grant No. SKLGP2012Z011) and the National Nature Science Foundation of China (Grant No. 40972190) for finance support. They also thank the Mapletrans Corporation for polishing this paper.

1. F. D. Patton, "Multiple modes of shear failure in rock," in: Proc. of the 1st Congr. of International Society of Rock Mechanics (Lisbon, Portugal, 1966), Vol. 1, pp. 509513.

2. H. Bock, "A simple failure criterion for rough joints and compound shear surfaces," Eng. Geol., 14, No. 4, 241-254 (1979).

3. E. Hoek, "Strength of jointed rock masses," Geotechnique, 33, No. 3, 187-223 (1983).

4. I. W. Johnston and T. S. K. Lam, "Shear behavior of regular triangular concrete/rock joints-analysis," J. Geotech. Eng., 115, No. 5, 711-727 (1989).

5. M. Bahaaddini, G. Sharrock, and B. K. Hebblewhite, "Numerical direct shear tests to model the shear behaviour of rock joints," Comput. Geotech., 51, 101-115 (2013).

6. M. Singh and B. Singh, "Modified Mohr-Coulomb criterion for nonlinear triaxial and polyaxial strength of jointed rocks," Int. J. Rock Mech. Min. Sci., 51, 43-52 (2012).

7. N. Barton, "Shear strength criteria for rock, rock joints, rockfill and rock masses: Problems and some solutions," J. Rock Mech. Geotech. Eng., 5, No. 4, 249-261 (2013).

8. L. Y. Zhang, "Estimating the strength of jointed rock masses," Rock Mech. Rock Eng., 43, No. 4, 391-402 (2010).

9. N. Barton, "Review of a new shear strength criterion for rock joints," Eng. Geol., 7, No. 4, 287-332 (1973).

10. G. Barla, F. Robotti, and L. Vai, "Revisiting large size direct shear testing of rock mass foundations," in: Proc. of the 6th Int. Conf. on Dam Engineering (Lisbon, Portugal, 2011), pp. 179-188.

11. D. R. Wines and P. A. Lilly, "Estimates of rock joint shear strength in part of the Fimiston open pit operation in Western Australia," Int. J. Rock Mech. Min. Sci., 40, No. 6, 929-937 (2003).

12. S. R. Hencher and L. Richards, "Laboratory direct shear testing of rock discontinuities," Ground Eng., 22, 24-31 (1989).

13. E. Hoek and J. W. Bray, Rock Slope Engineering, the 3rd edition, Institute of Mining and Metallurgy, London (1981).

14. J. Muralha, G. Grasselli, B. Tatone, et al., "ISRM suggested method for laboratory determination of the shear strength of rock joints, revised version," Rock Mech. Rock Eng., 47, No. 1, 291-302 (2014).

15. E. T. Brown (Ed), Rock Characterization, Testing and Monitoring - ISRM Suggested Methods, Pergamon Press, Oxford (1981), pp. 129-140.

16. G. Barla, M. Barla, and M. E. Martinotti, "Development of a new direct shear testing apparatus," Rock Mech. Rock Eng., 43, 117-122 (2010).

17. B. S. A. Tatone and G. Grasselli, "An investigation of discontinuity roughness scale dependency using high-resolution surface measurements," Rock Mech. Rock Eng., 46, No. 4, 657-681 (2013).

18. G. Grasselli, "Manuel Rocha medal recipient shear strength of rock joints based on quantified surface description," Rock Mech. Rock Eng., 39, No. 4, 295-314 (2006). 
19. C. C. Xia, Z. C. Tang, W. M. Xiao, and Y. L. Song, "New peak shear strength criterion of rock joints based on quantified surface description," Rock Mech. Rock Eng., 47, No. 2, 387-400 (2014).

20. M. K. Jafari, K. A. Hosseini, F. Pellet, et al., "Evaluation of shear strength of rock joints subjected to cyclic loading," Soil Dyn. Earthq. Eng., 23, No. 7, 619-630 (2003).

21. K. Kovari and A. Tisa, "Multiple failure state and strain controlled triaxial tests," Rock Mech., 7, 17-13 (1975).

22. M. R. Vergara, P. Kudella, and T. Triantafyllidis, "Large scale tests on jointed and bedded rocks under multi-stage triaxial compression and direct shear," Rock Mech. Rock Eng., 47, No. 2, 541-559 (2014).

Received 20. 10. 2014 